\title{
Uso de la realidad aumentada en la enseñanza-aprendizaje de ciencias naturales
}

\author{
Use of Augmented Reality in the Teaching-Learning \\ of Natural Science
}

\author{
Lilia Esther Muñoz-Arracera ${ }^{1} \bowtie$, Reyes Montenegro-Santos ${ }^{2}$ \\ ' Universidad Tecnológica de Panamá, Panamá \\ ${ }^{2}$ Universidad Tecnológica de Panamá, Panamá
}

Grupo de Investigación en Tecnologías Computacionales Emergentes, Facultad de Ingeniería de Sistemas Computacionales, Universidad Tecnológica de Panamá. Avenida Universidad Tecnológica de Panamá, Vía Puente Centenario. Apartado Postal 0819-07289. Email: lilia.munoz@utp.ac.pa

Recibido: 5 de septiembre del 2017 Aprobado: 30 de noviembre del 2017 Disponible en línea: 1 de enero del 2018

How to cite this article: L. E. Muñoz-Arracera, R. Montenegro-Santos, "Uso de la realidad aumentada en la enseñanzaaprendizaje de ciencias naturales", Revista Ingeniería Solidaria, vol. 14, no. 24, pp. 9, enero 2018. doi: https://doi. org/10.16925/in.v14i24.2155

\section{Resumen}

Introducción: el artículo es producto de la investigación "Uso de la realidad aumentada en la enseñanza-aprendizaje de ciencias naturales", desarrollada durante el 2016 y el primer semestre del 2017 en la Facultad de Ingeniería de Sistemas Computacionales de la Universidad Tecnológica de Panamá.

Problema: en Panamá no se brindan en la actualidad soluciones tecnológicas en el ámbito educativo que apoyen el proceso enseñanza-aprendizaje de sitios naturales y su conservación.

Objetivo: contribuir a mejorar el proceso enseñanza-aprendizaje de las ciencias naturales y la conservación del medio ambiente, por medio de dispositivos móviles y la realidad aumentada.

Metodología: se utiliza la investigación-acción, por la cual se planificó el trabajo a realizar, se desarrolló la aplicación, luego se observó a los estudiantes utilizar la aplicación y se plantearon los resultados de acuerdo con las observaciones y una rúbrica aplicada a los estudiantes.

Resultados: se desarrolló una aplicación con realidad aumentada que permite apoyar el proceso enseñanza-aprendizaje de ciencias naturales, y en este caso en particular de la enseñanza y conservación de sitios naturales.

Conclusión: se han logrado recrear animaciones que permiten a los estudiantes asimilar de forma efectiva la información de los sitios naturales, y así reforzar su aprendizaje a través de contenidos interactivos.

Originalidad: hasta el momento no se había abordado la implementación de este tipo de aplicaciones en escuelas rurales desde este enfoque. El proyecto ha permitido a la comunidad académica contar con una aplicación de gran valor que permite enseñar sobre la conservación de sitios naturales.

Limitaciones: el proyecto solo abarca el aprendizaje y la conservación de sitios naturales.

Palabras clave: aprendizaje, ciencias naturales, educación, Panamá, realidad aumentada. 


\title{
Use of Augmented Reality in the Teaching- Learning of Natural Science
}

\begin{abstract}
Introduction: The article derives from the research "Use of augmented reality in the teaching-learning of natural science" conducted in 2016 and the first half of 2017 at the School of Computer Systems Engineering, Universidad Tecnológica de Panamá.

Problem: Currently, Panama does not offer technological solutions in the educational field that support the process of teaching-learning about natural areas and their conservation.

Aim: To contribute to improving the process of teaching-learning natural science and the conservation of the environment through mobile devices and augmented reality.

Methods: Research-action was used, for which the work to be done was planned, the application was developed, students using the application were then observed, and the results were presented according to the observations and a rubric applied to students.
\end{abstract}

Results: An application with augmented reality was developed to support the process of teaching-learning natural science and, in this case, the teaching and conservation of natural areas.

Conclusion: We have managed to create animations that allow students to take in the information of natural areas and thus strengthen their learning through interactive contents.

Originality: So far, the implementation of this type of applications in rural schools had not been addressed from this perspective. The project has enabled the academic community to have a valuable application to teach about the conservation of natural areas.

Limitations: The project only covers the learning and conservation of natural areas.

Keywords: learning, natural science, education, Panama, augmented reality.

\section{Uso da realidade aumentada no ensino- aprendizagem de ciências naturais}

\section{Resumo}

Introdução: este artigo é produto da pesquisa "Uso da realidade aumentada no ensino-aprendizagem de ciências naturais", desenvolvida durante 2016 e o primeiro semestre de 2017, na Faculdade de Engenharia de Sistemas da Computação da Universidad Tecnológica de Panamá.

Problema: no Panamá, não são oferecidas, na atualidade, soluções tecnológicas no âmbito educativo que apoiem o processo ensino-aprendizagem de lugares naturais e sua conservação.

Objetivo: contribuir para melhorar o processo de ensino-aprendizagem das ciências naturais e a conservação do meio ambiente, por meio de dispositivos móveis e da realidade aumentada.

Metodologia: utilizou-se a pesquisa-ação, pela qual se planejou o trabalho a realizar; desenvolveu-se a aplicação; logo, os estudantes foram observados utilizando a aplicação e foram propostos os resultados de acordo com as observações e uma rubrica aplicada aos estudantes.

Resultados: desenvolveu-se uma aplicação com realidade aumenta que permite apoiar o processo ensino-aprendizagem de ciências naturais e, nesse caso em particular, do ensino e conservação de lugares naturais.

Conclusão: pôde-se recriar animações que permitissem os estudantes assimilarem de forma efetiva a informação dos lugares naturais e, assim, reforçarem sua aprendizagem por meio de conteúdos interativos.

Originalidade: até o momento, não se tem abordado a implementação desse tipo de aplicações em escolas rurais sob essa abordagem. 0 projeto permite à comunidade acadêmica contar como uma aplicação de grande valor que permite ensinar sobre a conservação de lugares naturais.

Limitações: 0 projeto somente abrange a aprendizagem e a conservação de lugares naturais.

Palavras-chave: aprendizagem, ciências naturais, educação, Panamá, realidade aumentada. 


\section{Introducción}

Con el pasar de los años la tecnología crece de manera progresiva, a tal punto que nuestras vidas cambian en relación con la forma de comunicarnos y relacionarnos con los demás, e incluso en la manera de interactuar con el medio en el que nos encontremos. Algunas tecnologías utilizadas en auge son los dispositivos móviles y la realidad aumentada (RA), los cuales permiten crear contenidos educativos capaces de apoyar el proceso enseñanza-aprendizaje. El concepto de RA nos lleva directamente hacia ese camino, debido a su relación con la tecnología y la forma en que esta puede enriquecer esa percepción de la realidad al potenciar cada uno de nuestros sentidos y ofrecer herramientas con las cuales la información del mundo real se complementa junto con la del mundo digital.

Cuando hablamos de RA hacemos referencia a una disciplina que se usa hace un tiempo en muchas áreas. El término se atribuye al investigador Tom Caudell [1], si bien a partir de la década de los sesenta ya se venían desarrollando interfaces que introducían conceptos de RA, lo cual ofrecía una infinidad de nuevas posibilidades de interacción en diferentes ámbitos. Existe una gran diversidad de aplicaciones que se pueden relacionar o establecer dentro de esta categoría, tales como: turismo, publicidad, aplicaciones industriales, dispositivos de navegación, arquitectura, simulación, entretenimiento y educación.

Los proyectos etiquetados como RA no han parado de crecer en los últimos años. Existe una multitud de definiciones de la RA y todas aportan algo interesante a la caracterización de este tipo de tecnología. Ronald Azuma [2] codificó las diferentes características que permiten su definición y diferenciación de la realidad virtual. Azuma considera la RA como aquella que permite al usuario ver en todo momento el mundo real, al que se le superponen objetos virtuales de manera que coexisten en el mismo espacio. Por su parte, Estebanell, Ferrés, Cornella y Codina [3] indican que las tecnologías en dispositivos móviles superan la limitación del tiempo y del espacio en los entornos de aprendizaje, pero además añaden que las aplicaciones de RA no solo responden a este tipo de exigencia, sino que la amplían de manera significativa al ofrecer información situada y contextualizada desde el lugar y en el momento que el consumidor la precisa. González [4] proporciona otras tres razones para apostar por la RA: (a) posibilita contenidos didácticos que son inviables de otro modo, (b) ayuda a que exista una continuidad en el hogar, y (c) aporta interactividad, experimentación, juego y colaboración.

El propósito de este proyecto consiste en lograr un enfoque educativo con el desarrollo de una aplicación móvil para la enseñanza y la conservación de sitios naturales de la provincia de Chiriquí, República de Panamá, lo cual permita a estudiantes de primaria en escuelas rurales obtener un aprendizaje enriquecido y conocer sobre la conservación de estos sitios; esto al brindarles nuevas tecnologías dentro de su entorno educacional a través de la implementación de RA. Se eligió este escenario de estudio, principalmente, en razón a la problemática que existe con relación a la conservación del medio ambiente y la necesidad de concientizar a las personas en etapas tempranas sobre su cuidado. En este sentido, la asignatura de ciencias naturales es un buen eje para el desarrollo del proyecto por el contenido que presenta.

El artículo está estructurado de la siguiente manera: en la segunda sección se presenta el trabajo relacionado con respecto al tema de estudio. La sección tres expone los métodos y materiales, mientras que en la cuarta se presentan los resultados obtenidos y la evaluación de la aplicación desarrollada. Finalmente, se describen las conclusiones y el trabajo futuro.

\section{Trabajos relacionados}

En los últimos años la RA ha aparecido con gran fuerza dentro de este contexto, ya que ofrece grandes posibilidades por su atractivo y capacidad de insertar objetos virtuales en un espacio real.

Una de las experiencias más recurrentes ha sido la basada en la metáfora del libro aumentado, empleada sobre todo en aplicaciones relacionadas con entornos educativos. Así, a partir de un marcador impreso en una de las páginas es posible acceder a información adicional mediante gráficos 3D que muestran figuras virtuales sobre las páginas del libro y se visualizan a través de la pantalla de un simple computador con una webcam. Uno de los primeros ensayos lo desarrolló el Human Interface Technology Laboratory de la Universidad de Washington cuando presentó el denominado "MagicBook", en el cual se evidenciaba el valor 
didáctico de esta tecnología y el gran atractivo que supone en contextos educacionales [5]. Por su parte, el proyecto de la empresa VirtualWare pretendía fomentar la utilización de materiales didácticos digitales para la materia de Conocimiento del Medio del tercer ciclo de educación primaria, a fin de que se utilizaran en el aula junto con el uso de los nuevos recursos docentes de reciente implantación, como son las pizarras digitales y las computadoras portátiles [6]. Álvarez et al. [7] utilizan la tecnología de RA a fin de implementar un recurso para la enseñanza de matemáticas y ciencias naturales. Su aplicación usa una cámara infrarroja para leer la superficie tridimensional de la arena y después dibujar sobre ella curvas de nivel y cuerpos de agua que se transforman cuando el usuario interactúa con la arena.

Por otro lado, en Panamá, se ha venido incorporando la RA en algunos contextos. Por ejemplo, en el 2013, se desarrolla "RA en la cinta costera", un proyecto que consistió en ofrecer un "tótem interactivo" que permitía a los usuarios seleccionar y visualizar diferentes partes de la cinta costera en 3D [8]. Otro de los proyectos en el 2015 fue "El Canal de Panamá en 3D y RA" [9], enfocado en el tercer juego de esclusas del Canal de Panamá; este proporciona un folleto interactivo que se activa con la aplicación Aurasma, la cual se puede instalar en tabletas y teléfonos inteligentes gracias a que conecta y combina la realidad física con la virtual. El folleto muestra videos sobre los aspectos más relevantes de la mayor obra de ingeniería civil del mundo y $3 \mathrm{D}$ animados con las enormes magnitudes del proyecto (dragado, excavado, hormigón y acero), así como un $3 \mathrm{D}$ de $360^{\circ}$ que permite acercarse, alejarse y girar, entre otras funciones. Sin embargo, a pesar de todo este desarrollo es poco lo que se ha logrado en el ámbito educativo en Panamá.

\section{Materiales y métodos}

En la última década, el uso de las Tic en la sociedad ha avanzado de forma progresiva y con resultados satisfactorios. Una de las áreas en la que estas tecnologías están revolucionando el concepto clásico es la educativa. En este sentido, una de las tecnologías que puede suponer una innovación en las aulas es la RA. Esta técnica permite integrar modelos virtuales $3 \mathrm{D}$ a la realidad física mediante un dispositivo y un computador [10]. A continuación, se describen los métodos y materiales utilizados en el desarrollo de este proyecto.

\subsection{Descripción de la problemática}

El auge de las TIC a nivel mundial crece constantemente y aprovecha sus beneficios en la educación, de modo que las integra de distintas formas y en diversas asignaturas. Hoy, con la disposición de implementar RA, cambia aún más la manera en que se transmiten los conocimientos dentro de las aulas de clases, sin embargo, en Panamá no se brindan soluciones tecnológicas basadas en RA para el ámbito educativo, y mucho menos dirigidas a escuelas ubicadas en zonas rurales.

Por su parte, el propósito de la enseñanza de las ciencias naturales en la escuela es favorecer la alfabetización científica de los ciudadanos desde la escolaridad temprana, en procura de que comprendan conceptos, practiquen procedimientos y desarrollen actitudes que les permitan participar de una cultura analítica y crítica ante la información emergente.

En este contexto, la provincia de Chiriquí cuenta con una gran variedad de flora y fauna debido a la cantidad de sitios naturales que posee, pero a medida que pasan los años algunos de estos sitios se ven afectados, principalmente, a causa de las diversas actividades humanas. Esto genera grandes afectaciones al medio ambiente. Aunque se encuentran sitios que aún se mantienen protegidos y se beneficia así su conservación, no escapan de esta realidad, ya que si no se realizan esfuerzos por crear conciencia en las personas pueden verse igualmente afectados en un futuro, al igual que se pueden incrementar las afectaciones en sitios que ya han sufrido daños. Para el desarrollo de este proyecto se han visitado algunos sitios de la provincia, entre estos el río Chico, el cual se ha visto deteriorado por actividades humanas, y los Cangilones de Gualaca que se mantiene como un sitio turístico de gran valor.

La contaminación es un cambio perjudicial en las características físicas, químicas o biológicas del aire, la tierra e incluso el agua, lo que puede afectar de forma nociva la vida humana o la de otras especies, las condiciones de vida del ser humano y deteriorar los recursos naturales renovables [11]. Al ser la contaminación un factor alarmante dentro de la sociedad, la aplicación de RA que se busca desarrollar e implementar se enfoca en la conservación 
de los sitios naturales, ya que el nivel de contaminación y deterioro de los recursos naturales en Panamá está ocasionando grandes daños —así como en el resto de los países del mundo- a causa de la destrucción de los bosques, lo que ha generado la extinción de plantas y animales.

\subsection{Propuesta}

Se busca desarrollar una aplicación para dispositivos móviles cuyo objetivo principal es concientizar en etapas tempranas a los niños sobre la conservación del medio ambiente. Para esto se empleará la $\mathrm{RA}$, ya que es una tecnología que brinda el aspecto interactivo, además de apoyar el proceso enseñanza-aprendizaje en la asignatura de ciencias naturales. Se espera contar con los siguientes resultados:

- Una plataforma para dispositivos móviles que contenga la información virtual de los sitios naturales seleccionados y su conservación.

- Proporcionar láminas didácticas en las que se ilustre cada sitio elegido y se exponga su información y pasos a seguir, con la finalidad de mantener estos sitios conservados.

- Lograr una interacción virtual satisfactoria por parte del usuario al momento de visualizar la lámina que se entrega junto con la aplicación de RA desarrollada.

- Despertar una cultura de conservación en los estudiantes que utilizan la plataforma, a fin de aportar así dentro de su proceso de aprendizaje de la asignatura de ciencias naturales y los recursos tecnológicos.

\section{Resultados}

En el marco de los resultados de este proyecto se ha desarrollado una aplicación móvil. La arquitectura de la aplicación se puede apreciar en la figura 1.

A continuación, se describen algunos de los componentes de la arquitectura de la aplicación que se desarrolló con la herramienta Vuforia:

- Cámara. Esta instancia se encarga de que cada fotograma capturado por la cámara se transfiera de manera eficiente al rastreador (tracker).

- Rastreador. Contiene los diferentes algoritmos de visión computacional que permiten detectary seguir cada uno de los objetos en los fotogramas capturados. Basados en la imagen tomada por la cámara, diferentes algoritmos se encargan de detectar las imágenes de referencia.

- Estado de objetos. Es donde se almacenan los resultados obtenidos por el rastreador para que los utilice el procesador de video.

- Procesador de video. Esta instancia procesa la imagen capturada que se encuentra almacenada en el estado de objetos. El rendimiento del renderizado del video varía dependiendo del dispositivo.

- Convertidor de imagen. La instancia conversor formato de pixel realiza la conversión del formato con el que trabaja la cámara a un formato adecuado para el renderizado y el seguimiento. Implica un submuestreo cuyo fin es obtener la imagen capturada por la cámara en diferentes resoluciones, disponibles en la pila de fotogramas convertidos.

\subsection{Láminas}

Las láminas que se elaboraron en el proyecto contienen imágenes seleccionadas de los sitios que se eligieron y visitaron. Dentro de la estructura general de las láminas se tiene que resaltar principalmente el nombre del sitio que se muestra en cada una de ellas. En la figura 2 se puede apreciar la lámina de los Cangilones de Gualaca, ubicados en el distrito de Gualaca.

El usuario podrá obtener de las láminas información de cada sitio, al igual que las prácticas de conservación que se deben realizar a fin de mantenerlos preservados; también se incluyen aspectos sobre afectaciones que se pueden dar por causa de las actividades humanas.

Al momento que el usuario enfoque la cámara de su dispositivo sobre la lámina didáctica podrá interactuar en tiempo real con el contenido de RA que le proporcionará la aplicación, todo esto con el propósito de apoyar tecnológicamente la enseñanza de ciencias naturales y los cuidados al medio ambiente.

\subsection{Interfaz de la aplicación}

La interfaz de la aplicación de RA es una pantalla sencilla que permite al usuario elegir, dar inicio o salir de la aplicación y obtener información acerca de la RA. 


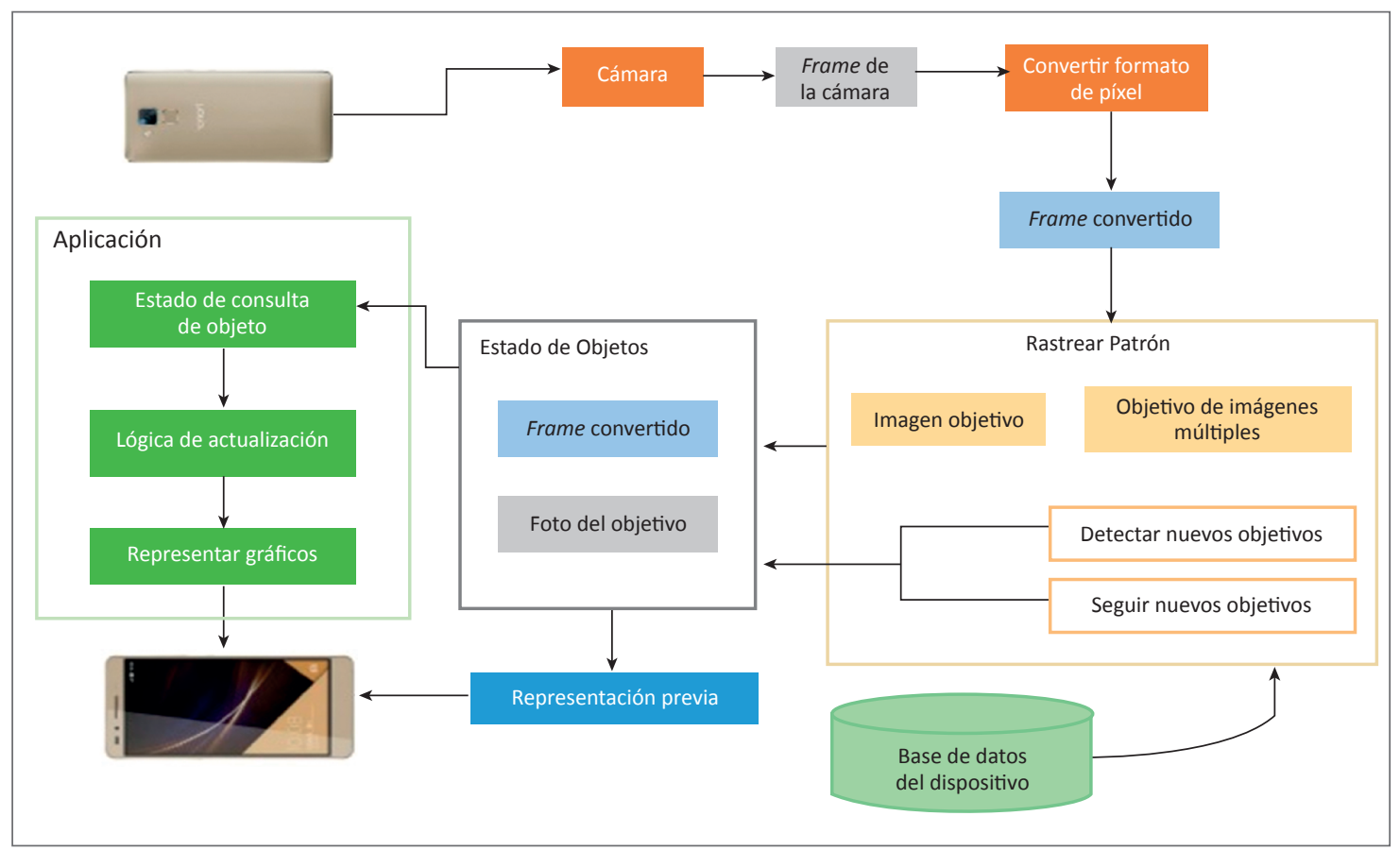

Figura 1. Arquitectura de la aplicación

Fuente: elaboración propia

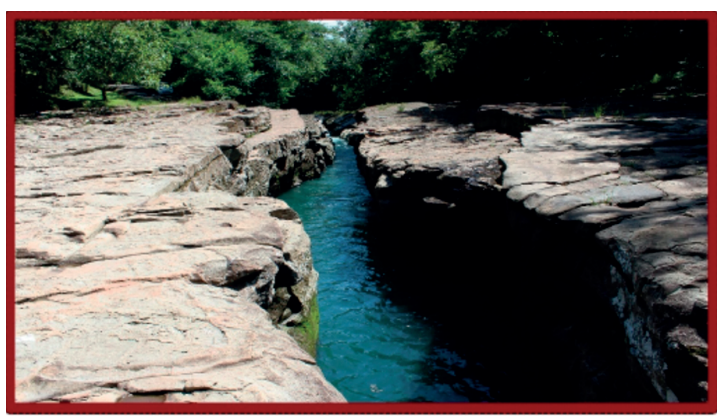

LOS CANGILONES DE G U A L A C A

Figura 2. Lámina de los Cangilones de Gualaca Fuente: elaboración propia

Una vez el usuario decide iniciar la sesión se podrá apreciar en la pantalla del móvil la entrada de imágenes mostradas a través de la cámara, de manera que al momento de la detección de un patrón almacenado en la base de datos se despliega la animación correspondiente, lo que permite al usuario interactuar y aprender. La opción "Salir" permite salir de la aplicación, y la opción "Acerca de la RA" permite visualizar el objetivo por el cual se ha creado la aplicación y brinda una breve descripción de lo que es la RA. En la figura 3 se puede apreciar la interfaz de la aplicación.

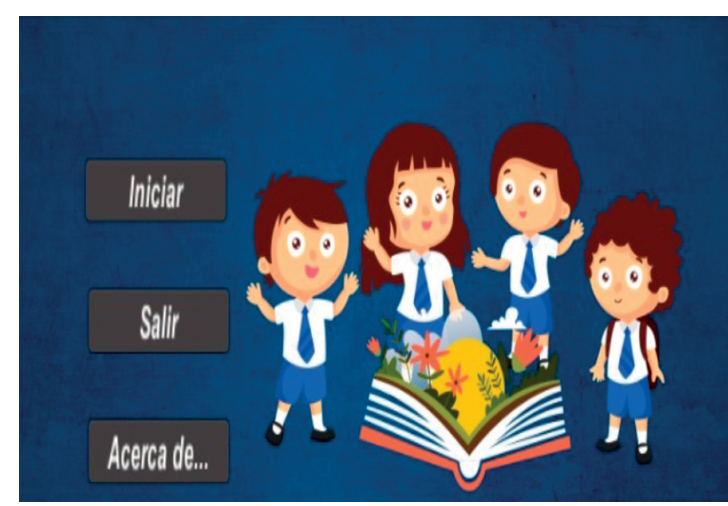

Figura 3. Menú principal de la aplicación Fuente: elaboración propia

\subsection{Interacción}

La interacción del usuario con la aplicación se realizó por completo a través de las láminas didácticas, las cuales contienen las imágenes de los sitios naturales que se utilizaron para el proyecto. Estas 
se presentan ante la cámara del dispositivo móvil para así obtener todo el contenido de RA que tiene asociado dentro de la aplicación.

A fin de obtener correctamente las animaciones $3 \mathrm{D}$ el usuario mantiene la lámina frente a la cámara - debe evitar taparla con algún objetocon la finalidad de que el patrón a reconocer se mantenga visible siempre que esté en uso. En caso de que el usuario ya no desee que se muestre la animación solo debe dejar de enfocar la lámina con la cámara, o si desea interactuar con otro sitio solo debe mover el dispositivo y enfocar otra lámina. En la figura 4 se puede apreciar a uno de los estudiantes utilizando la aplicación.

La aplicación de RA tiene como propósito facilitar en todo momento la interacción del usuario con el contenido virtual y las láminas, a fin de evitar confusiones sobre su utilización y proporcionarle una herramienta de fortalecimiento educativo en torno a la enseñanza en ciencias naturales sobre la conservación mediante un manejo sencillo de la herramienta, al igual que con un funcionamiento interactivo y atractivo.

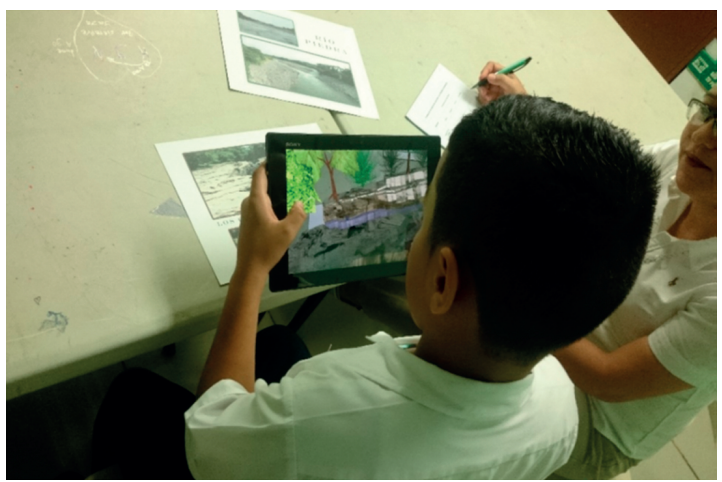

Figura 4. Estudiante probando la herramienta

Fuente: elaboración propia

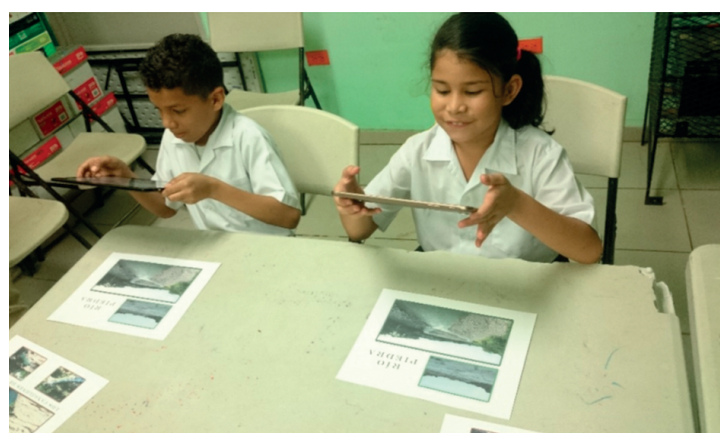

Figura 5. Estudiantes probando la herramienta Fuente: elaboración propia

\subsection{Evaluación de la aplicación}

Para la evaluación de la aplicación desarrollada se solicitó autorización a la dirección del plantel escolar Escuela Barrio Lassonde. La dirección nos asignó a la docente que impartía clases en tercer grado. A ella se le explicó en qué consistía el proyecto, se le instruyó en el uso de la aplicación; ella se encargó de seleccionar a 20 estudiantes cuyas edades oscilaban entre los ocho y los nueve años de edad. La finalidad de esta etapa era validar el funcionamiento de la aplicación. Los alumnos utilizaron la aplicación y respondieron a preguntas acerca de la funcionalidad, el diseño y el contenido.

La evaluación de la aplicación determinó que los estudiantes se adaptaron de una manera buena al uso de la aplicación. No fue de manera excelente, ya que la organización de la información en las láminas en primera instancia no fue la adecuada. Cuando se desarrollaron las láminas se colocaron tres imágenes del sitio en cada una, pero solo la imagen principal de mayor tamaño realizaba la activación del contenido 3D; las otras dos estaban como referencia a diferentes vistas del sitio a mostrar, lo cual - consideramos- confundía al estudiante, por lo que se realizaron cambios en las láminas y se dejó solo una imagen por lámina.

En cuanto a la facilidad de la navegación, la comprensión y la satisfacción del usuario, los resultados fueron excelentes, lo que nos permite evidenciar que la aplicación desarrollada permite, de una manera sencilla, enseñar al estudiante conceptos y visualizar cómo son los sitios naturales de una manera más real. En la figura 5 se pueden observar los resultados obtenidos en la evaluación.

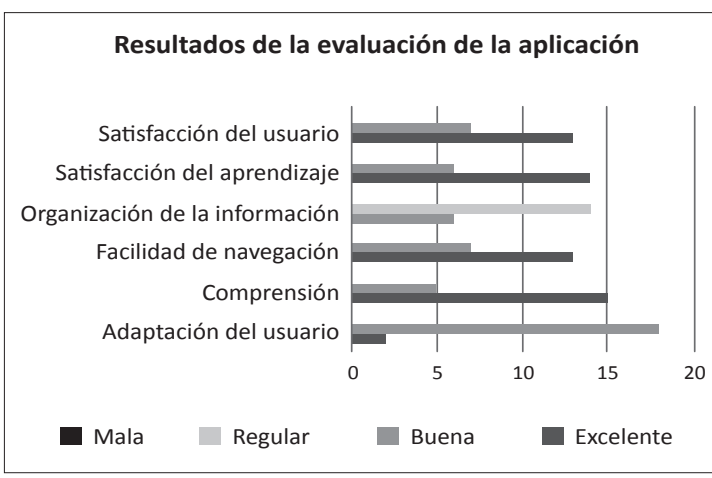

Figura 6. Resultados de la evaluación

Fuente: elaboración propia 


\section{Discusión}

Hoy en día, las exigencias en cuanto al uso de las TIC han aumentado a tal punto que se deben aplicar constantemente estrategias novedosas, aún más en el ámbito educativo, a fin de mejorar con esto la forma de atraer la atención de los estudiantes y obtener así un mejor rendimiento [12]. Esto es posible con la ayuda de plataformas flexibles que cuenten con la visualidad correcta, de manera que se realice esa síntesis adecuada de toda la información que se busca transmitir. Es en esto que la RA se transforma en una herramienta importante, ya que lleva a una provocación visual sin dejar de lado la importancia del contenido a mostrar.

En la actualidad, el desarrollo de proyectos en entornos de RA se lleva a cabo en casi todos los ámbitos del saber. El entorno educativo no escapa de esta realidad.

Existe una mejora significativa en los procesos de aprendizaje gracias a la RA, ya que la motivación de los alumnos se ve incrementada al incorporar contenidos 3D en los materiales didácticos. Esta tecnología potencia el efecto educativo al ofrecer actividades de entretenimiento $y$, a su vez, demuestra que es una gran herramienta que motiva a los estudiantes. Al obtener los beneficios antes mencionados este proyecto sirve para mejorar la manera tradicional en que se imparten las clases en las aulas escolares aplicado al área de las ciencias naturales, de manera que se tornan más atractivas mediante la implementación de una nueva tecnología que, poco a poco, se va transformando en un instrumento útil y de gran importancia para la enseñanza y nos ayuda a inculcar en los alumnos ese conocimiento que muchas veces desconocen sobre los sitios naturales que los rodean. De igual forma, da a conocer en etapas tempranas las prácticas adecuadas para su conservación.

El proyecto se desarrolla para escuelas de áreas rurales y la principal limitante encontrada fue la carencia de aplicaciones con las cuales los estudiantes pudieran interactuar.

El trabajo aquí expuesto tiene como objetivo primordial brindar una herramienta que facilite el proceso enseñanza-aprendizaje efectivo en ciencias naturales, particularmente en la conservación del medio ambiente. La herramienta permite interactuar con cada uno de los elementos en un plano tridimensional atractivo mediante láminas, en el cual se brinda información relevante sobre los sitios naturales y las instrucciones para el cuidado de estos.

Los beneficios y los aportes que esta investigación puede generar son importantes retos que exigen: (a) acercar a los estudiantes en etapas tempranas al cuidado y protección del medio ambiente, (b) reconocer que la RA motiva al estudiante y permite que su aprendizaje sea más efectivo, y (c) promover el uso de la RA para el desarrollo de aplicaciones en otros escenarios educativos relacionados con matemáticas, español, etc.

\section{Conclusiones}

Mediante el diseño y desarrollo de escenarios para la enseñanza y la conservación de sitios naturales a través de la RA, se han logrado recrear animaciones que permiten a los estudiantes asimilar de forma efectiva la información de los sitios, y reforzar así su aprendizaje a través de contenidos interactivos que generan una mayor motivación por aprender. De esta manera, la creación de las diferentes láminas didácticas fue necesaria ya que les permite vivir esa experiencia de interactuar con varios contenidos virtuales. Gracias a su uso, es posible optimizar los procesos de aprendizaje y aumentar el interés y la participación de los estudiantes.

Por ser una aplicación autocontenida, se debe contar con un sistema de alojamiento externo, a fin de que el consumo de almacenamiento en los dispositivos en los que se va a instalar se reduzca $y$, de esta forma, también se puedan añadir nuevos modelos y patrones sin la necesidad de compilar y generar un instalador cada vez que se realice algún cambio.

Uno de los aspectos importantes a considerar en el desarrollo de este tipo de aplicación es la cantidad de recursos que consumen los contenidos 3D a desplegar. En este sentido, se deben considerar medidas que permitan optimizar dicho contenido para que el rendimiento y la compatibilidad con dispositivos móviles de gama media-baja se pueda llevar a cabo de manera exitosa.

Como trabajo futuro se pretende implementar la aplicación desarrollada en una mayor cantidad de centros educativos a nivel de primaria, con el propósito de que más estudiantes y docentes se vean beneficiados con este tipo de herramientas, así como utilizar esta aplicación como base con miras hacia el desarrollo e implementación en diferentes 
áreas de la educación. Otro de los aspectos a considerar como trabajo futuro es tener en cuenta otros sitios naturales para el desarrollo de más láminas y definir otras variables que permitan evaluar más escenarios con los estudiantes.

\section{Agradecimientos}

Agradecemos al Sistema Nacional de Investigación por el financiamiento para el desarrollo del proyecto; a los estudiantes y profesores de la Escuela Barrio Lassonde por el apoyo que nos brindaron.

\section{Referencias}

[1] T. Caudell y D. Mizell, "Augmented reality: an application of heads-up display technology to manual manufacturing processes", en International Conference on System Sciences, vol. 2, Kauai, Hawái: 1992, pp. 659-669. doi: https://doi.org/10.1109/ HICSS.1992.183317

[2] R. Azuma, "A survey of augmented reality", Presence: Teleoperators and Virtual Environments, vol. 6, no. 4, pp. 355-385, 1997. doi: https://doi.org/10.1162/ pres.1997.6.4.355

[3] M. Estebanell, J. Ferrés, P. Cornella y D. Codina, "Realidad aumentada y códigos QR en educación", en J. Hernández, M. Pennesi, D. Sobrino yA. Vázquez (coords.), Tendencias emergentes en educación con TIC, Barcelona, Editorial Espiral: 2012, pp. 277-320. [En línea]. Disponible en: https://dialnet.unirioja. es/ servlet/articulo? codigo $=4230624$

[4] O. González, "Educación aumentada”, Boletín del Centro de Conocimiento de Tecnologías Aplicadas a la Educación (CITA), vol. 19, mar. 2001,p. 1. [En línea]. Disponible en http://www.elblogdeoscargonzalez. es/2013/03/educacion-aumentada.html
[5] M. Billinghurst, H. Kato e I. Poupyrev, "The MagicBook: a transitional AR interface. Elsevier Computers and Graphics, vol. 25, pp. 745-753, 2001. doi: https:// doi.org/10.1016/S0097-8493(01)00117-0

[6] VirtualWare, "Comienza el despliegue tecnológico del programa Eskola 2.0”, pp. 1-2, 2010. [En línea]. Disponible en: http://www.virtualware.es/es/noticia/actualidad-virtualware/comienza-el-despliegue -tecnologico-del-programa-eskola-2-0.aspx

[7] S. Álvarez, L. Delgado, M. González, T. Martín, F. Almaraz y C. Ruiz, "El Arenero Educativo: la realidad aumentada un nuevo recurso para la enseñanza", Revista de Educación Mediática y TIC, vol. 6., no. 1, pp. 105-123, 2017. [En línea]. Disponible en: https://www.uco.es/ucopress/ojs/index.php/ edmetic/article/view/ 5810

[8] Innovae Vision, "Realidad Aumentada en la Cinta Costera Panamá”, pp. 1-33. 2013. [En línea]. Disponible en: https://www.youtube.com/watch?v= $5 \mathrm{DkpW}$-is-8

[9] J. Hernández, M. Pennesi, D. Sobrino y A. Vázques. Tendencias emergentes en Educación con TICM, España, Asociación Espiral: 2012, pp. 288. [En línea]. Disponible en: https:/ciberespiral.org/tendencias/ Tendencias_emergentes_en_educacin_con_TIC.pdf

[10] R. Gallego, N. Saura y P. Nuñez, “AR-Learning: libro interactivo basado en realidad aumentada con aplicación a la enseñanza”, no. 8 extra, pp. 74-88, 2012. [En línea]. Disponible en: https://dialnet.unirioja. es/servlet/articulo? codigo $=5385923$

[11] R. González y M. González, "Efectos de la política comercial en los recursos naturales y el ambiente", Revista Internacional Contaminación Ambiente, vol. 26, no. 2, pp. 151-163, 2010. [En línea]. Disponible en: http://www.scielo.org.mx/ pdf/rica/v26n2/ v26n2a6.pdf

[12] I. de la Horra, "Realidad Aumentada, una evolución educativa”, Revista de Educación Mediática y TIC, vol. 1, no. 6, pp. 9-21, 2016. [En línea]. Disponible en: https://www.uco.es/ucopress/ojs/index.php/edmetic/article/view/5762 